\title{
Integro-differential closure equations for inhomogeneous turbulence
}

\author{
T. J. O'Kane* J. S. Frederiksen ${ }^{\dagger}$
}

(Received October 2001; revised June 2002)

\begin{abstract}
We present numerical and computational techniques to solve systems of integro-differential closure equations for inhomogeneous two-dimensional turbulent flow. The closure equations, representing the first tractable closure theory for inhomogeneous flow over mean (single realization) topography, are based on a quasi-diagonal direct interaction approximation derived via renormalization techniques. The equations are computationally challenging due to the potentially long time history integrals. In order to reduce the computational cost we have implemented a formal restart procedure for the two and three point cumulant terms. The restart procedure is shown to be in good agreement with the closure without restarts and results are compared to direct numerical simulation of the barotropic vorticity equation.
\end{abstract}

*School of Mathematical Sciences, Monash University, Clayton, Australia. mailto: Terence.0'Kane@csiro.au

${ }^{\dagger}$ CSIRO Atmospheric Research, Aspendale, Australia. mailto: Jorgen.Frederiksen@csiro.au

${ }^{0}$ See http: //anziamj . austms.org.au/V44/CTAC2001/0kan for this article, (c) Austral. Mathematical Soc. 2003. Published 1 April 2003. ISSN 1446-8735 


\section{Contents}

1 Introduction

C570

2 Two-dimensional flow over topography

C572

3 CUQDIA closure equations

C574

4 Restart terms

C579

5 Numerical methods

C582

6 Comparison of QDIA and CUQDIA with DNS

C583

7 Conclusion

C586

A 2-point restart terms

C587

References

C588

\section{Introduction}

In this paper, we describe the numerical implementation of the quasi-diagonal direct interaction approximation (QDIA) closure theory of Frederiksen [1]. The QDIA closure is the first tractable closure theory for general inhomogeneous flow over mean (single realization) topography. It was formulated with the aim of establishing a general expression for the eddy-topographic force which parametrizes the interaction between retained scale topography with subgrid-scale eddies. The importance of parameterizing the eddy-topographic force for obtaining realistic ocean circulations has been shown by Holloway [2], but a rigorous theory of the parametrization had been lacking prior to the development of the QDIA closure theory. 
Here we implement the QDIA closure and compare its performance with the statistics of ensemble averaged direct numerical simulations (DNS) for two-dimensional Navier-Stokes flows over topography. The QDIA closure incorporates prognostic equations for the mean vorticity, for the vorticity covariance and for response functions. Both the closure and DNS are formulated for flows on the doubly periodic domain and in this article we focus on low-order truncations of the equations. The QDIA consists of a set of integrodifferential closure equations with potentially long time-history integrals that can result in a large computational task for long integrations.

We also present a variant of the QDIA closure which has similar accuracy to the QDIA but which is computationally much more efficient. This variant, termed the cumulant update quasi-diagonal direct interaction approximation (CUQDIA), overcomes the problem of the long time-history integrals by periodically truncating them, calculating both the two- and three-point cumulants and then uses these as new non-Gaussian initial conditions for subsequent integrations. Our method is a generalization of a cumulant update scheme for the homogeneous DIA closure proposed by Rose [3] and applied to a three component problem in plasma physics. It has also been extensively applied to non-Markovian closures for two-dimensional isotropic turbulence by Frederiksen et al. [4] and Frederiksen and Davies [5].

The QDIA closure, which is based on renormalized-perturbation theory, preserves conservation of kinetic energy and potential enstrophy and guarantees realizability for the diagonal elements of the covariance matrices. Here we compare the performance of the QDIA and CUQDIA closures with DNS for forced dissipative flows over topography in 20 day simulations; we employ typical meteorological space and time scales in our numerical experiments. 
We present the equations for two-dimensional barotropic flow over topography on an $f$-plane and for doubly periodic boundary conditions in Section 2. The quasi-diagonal direct interaction equations, with cumulant update restarts are summarized in Section 3. Section 4 shows that we only need to include the 2- and 3-point cumulants in the restart procedure to be consistent with the approximations in the QDIA closure. The numerical methods used to solve the CUQDIA closure and DNS equations are summarized in Section 5 while in Section 6 we compare the performance of the QDIA and CUQDIA closures with DNS for forced dissipative flows over topography. The conclusions are presented in Section 7, and the Appendix A formulates the expression for the 2-point restart terms.

\section{Two-dimensional flow over topography}

The evolution equation for two-dimensional flow over a fixed topography on a periodic $f$-plane $(0 \leq x, y \leq 2 \pi)$ is the non-dimensional barotropic vorticity equation

$$
\frac{\partial \zeta}{\partial t}=-J(\psi, \zeta+h)+\nu_{0} \nabla^{2} \zeta+f^{0}
$$

where $f^{0}$ and $\nu_{0}$ are the bare forcing and bare viscosity respectively. The vorticity is given in terms of the stream function

$$
\zeta=\nabla^{2} \psi
$$

We write (1) in spectral form via

$$
\zeta(\mathbf{x}, t)=\sum_{\mathbf{k}} \zeta_{\mathbf{k}}(t) \exp i \mathbf{k} \cdot \mathbf{x}
$$


where

$$
\zeta_{\mathbf{k}}(t)=\frac{1}{(2 \pi)^{2}} \int_{0}^{2 \pi} d^{2} \mathbf{x} \zeta(\mathbf{x}, t) \exp (-i \mathbf{k} \cdot \mathbf{x}),
$$

and $\mathbf{x}=(x, y), \mathbf{k}=\left(k_{x}, k_{y}\right)$. The spectral vorticity equation is

$$
\begin{aligned}
& \left(\frac{\partial}{\partial t}+\nu_{0}(k) k^{2}\right) \zeta_{\mathbf{k}}(t)=\sum_{\mathbf{p}} \sum_{\mathbf{q}} \delta(\mathbf{k}+\mathbf{p}+\mathbf{q}) \\
& \quad \times\left[K(\mathbf{k}, \mathbf{p}, \mathbf{q}) \zeta_{-\mathbf{p}} \zeta_{-\mathbf{q}}+A(\mathbf{k}, \mathbf{p}, \mathbf{q}) \zeta_{-\mathbf{p}} h_{-\mathbf{q}}\right]+f_{\mathbf{k}}^{0}
\end{aligned}
$$

where $k=\left(k_{x}^{2}+k_{y}^{2}\right)^{1 / 2}$ and $\zeta_{-\mathbf{k}}=\zeta_{\mathbf{k}}^{*}$ are conjugate.

The interaction coefficients are governed by the following relationships

$$
\begin{aligned}
A(\mathbf{k}, \mathbf{p}, \mathbf{q}) & =-\left(p_{x} q_{y}-p_{y} q_{x}\right) / p^{2} \\
K(\mathbf{k}, \mathbf{p}, \mathbf{q}) & =\frac{1}{2}[A(\mathbf{k}, \mathbf{p}, \mathbf{q})+A(\mathbf{k}, \mathbf{q}, \mathbf{p})] \\
& =\frac{1}{2}\left(p_{x} q_{y}-p_{y} q_{x}\right)\left(p^{2}-q^{2}\right) / p^{2} q^{2}
\end{aligned}
$$

where

$$
K(\mathbf{k}, \mathbf{p}, \mathbf{q})+K(\mathbf{p}, \mathbf{q}, \mathbf{k})+K(\mathbf{q}, \mathbf{k}, \mathbf{p})=0 .
$$

Also $\delta$ is the Kronecker delta function.

For an ensemble of flows satisfying (5) we express the vorticity for a given realization in terms of the ensemble mean $\left\langle\zeta_{\mathbf{k}}\right\rangle$ and the deviation from the ensemble mean $\hat{\zeta}_{\mathbf{k}}$ :

$$
\zeta_{\mathrm{k}}=\left\langle\zeta_{\mathrm{k}}\right\rangle+\hat{\zeta}_{\mathrm{k}}
$$


Hence we write these equations for the ensemble mean

$$
\begin{aligned}
& \left(\frac{\partial}{\partial t}+\nu_{0}(k) k^{2}\right)\left\langle\zeta_{\mathbf{k}}\right\rangle \\
& =\sum_{\mathbf{p}} \sum_{\mathbf{q}} \delta(\mathbf{k}+\mathbf{p}+\mathbf{q}) K(\mathbf{k}, \mathbf{p}, \mathbf{q})\left[\left\langle\zeta_{-\mathbf{p}}\right\rangle\left\langle\zeta_{-\mathbf{q}}\right\rangle+C_{-\mathbf{p}-\mathbf{q}}(t, t)\right] \\
& +\sum_{\mathbf{p}} \sum_{\mathbf{q}} \delta(\mathbf{k}+\mathbf{p}+\mathbf{q}) A(\mathbf{k}, \mathbf{p}, \mathbf{q})\left\langle\zeta_{-\mathbf{p}}\right\rangle h_{-\mathbf{q}}+\left\langle f_{\mathbf{k}}^{0}\right\rangle
\end{aligned}
$$

and the deviation

$$
\begin{aligned}
& \left(\frac{\partial}{\partial t}+\nu_{0}(k) k^{2}\right) \hat{\zeta}_{\mathbf{k}} \\
& =\sum_{\mathbf{p}} \sum_{\mathbf{q}} \delta(\mathbf{k}+\mathbf{p}+\mathbf{q}) K(\mathbf{k}, \mathbf{p}, \mathbf{q})\left[\left\langle\zeta_{-\mathbf{p}}\right\rangle \hat{\zeta}_{-\mathbf{q}}\right. \\
& \left.\quad+\hat{\zeta}_{-\mathbf{p}}\left\langle\zeta_{-\mathbf{q}}\right\rangle+\hat{\zeta}_{-\mathbf{p}} \hat{\zeta}_{-\mathbf{q}}-C_{-\mathbf{p}-\mathbf{q}}(t, t)\right] \\
& +\sum_{\mathbf{p}} \sum_{\mathbf{q}} \delta(\mathbf{k}+\mathbf{p}+\mathbf{q}) A(\mathbf{k}, \mathbf{p}, \mathbf{q}) \hat{\zeta}_{-\mathbf{p}} h_{-\mathbf{q}}+\hat{f}_{\mathbf{k}}^{0}
\end{aligned}
$$

with $f_{\mathbf{k}}^{0}=\left\langle f_{\mathbf{k}}^{0}\right\rangle+\hat{f}_{\mathbf{k}}^{0}$ and the single time cumulant in (10) and (11) determined by

$$
C_{-\mathbf{p}-\mathbf{q}}(t, s)=\left\langle\hat{\zeta}_{-\mathbf{p}}(t) \hat{\zeta}_{-\mathbf{q}}(s)\right\rangle
$$

\section{CUQDIA closure equations}

The derivation of the closure equations, without restarts, can be found in Frederiksen [1] and we therefore only present a summary of the results here. In order to obtain the closure equations the vorticity is expanded in a perturbation series to find the zeroth order 
and first order terms. We write the solution to the first order equation for $\hat{\zeta}_{\mathrm{k}}^{(1)}$ in terms of the response or Greens function $R_{\mathrm{k}}^{(0)}(t, s)$, and express the 2-time cumulant to first order.

We assume that the initial $\hat{\zeta}_{\mathbf{k}}^{(1)}\left(t_{0}\right)$ have a multivariate Gaussian distribution so that the initial covariance matrix is diagonal. As well, we suppose that the off-diagonal elements of the 2-time covariance and response function matrices are small compared to the diagonal elements. After renormalization [1] we find that the offdiagonal 2-point cumulant is written in terms of diagonal cumulant and response functions:

$$
\begin{aligned}
& C_{\mathbf{k}-\mathbf{l}}(t, \hat{t})=\int_{t_{0}}^{t} d s R_{\mathbf{k k}}(t, s) C_{\mathbf{l}-\mathbf{l}}(s, \hat{t}) \\
& \quad \times\left[A(\mathbf{k},-\mathbf{l}, \mathbf{l}-\mathbf{k}) h_{(\mathbf{k}-\mathbf{l})}+2 K(\mathbf{k},-\mathbf{l}, \mathbf{l}-\mathbf{k})\left\langle\zeta_{(\mathbf{k}-\mathbf{l})}(s)\right\rangle\right] \\
& +\int_{t_{0}}^{t} d s R_{-\mathbf{l}-\mathbf{l}}(\hat{t}, s) C_{\mathbf{k}-\mathbf{k}}(t, s) \\
& \quad \times\left[A(-\mathbf{l}, \mathbf{k}, \mathbf{l}-\mathbf{k}) h_{(\mathbf{k}-\mathbf{l})}+2 K(-\mathbf{l}, \mathbf{k}, \mathbf{l}-\mathbf{k})\left\langle\zeta_{(\mathbf{k}-\mathbf{l})}(s)\right\rangle\right]
\end{aligned}
$$

In a similar way, the off-diagonal response function

$$
\begin{aligned}
& R_{\mathbf{k} \mathbf{l}}(t, \hat{t})=\int_{\dot{t}}^{t} d s R_{\mathbf{k k}}(t, s) R_{\mathbf{l}}(s, \hat{t}) \\
& \quad \times\left[A(\mathbf{k},-\mathbf{l}, \mathbf{l}-\mathbf{k}) h_{(\mathbf{k}-\mathbf{l})}+2 K(\mathbf{k},-\mathbf{l}, \mathbf{l}-\mathbf{k})\left\langle\zeta_{(\mathbf{k}-\mathbf{l})}(s)\right\rangle\right]
\end{aligned}
$$

The end result of this procedure is the determination of the single time cumulant and the diagonal 2-time cumulant. Hence, from (10) 
we write equations for the mean-field

$$
\begin{aligned}
& \left(\frac{\partial}{\partial t}+\nu_{0}(k) k^{2}\right)\left\langle\zeta_{\mathbf{k}}\right\rangle=\sum_{\mathbf{p}} \sum_{\mathbf{q}} \delta(\mathbf{k}+\mathbf{p}+\mathbf{q}) \\
& \times\left[K(\mathbf{k}, \mathbf{p}, \mathbf{q})\left\langle\zeta_{-\mathbf{p}}(t)\right\rangle\left\langle\zeta_{-\mathbf{q}}(t)\right\rangle+A(\mathbf{k}, \mathbf{p}, \mathbf{q})\left\langle\zeta_{-\mathbf{p}}(t)\right\rangle h_{-\mathbf{q}}\right. \\
& \left.\quad+K(\mathbf{k}, \mathbf{p}, \mathbf{q}) \tilde{K}_{-\mathbf{p},-\mathbf{q}}^{(2)}(0,0) R_{-\mathbf{p}}(t, 0) R_{-\mathbf{q}}(t, 0)\right] \\
& \quad-\int_{t_{0}}^{t} d s \eta_{\mathbf{k}}(t, s)\left\langle\zeta_{\mathbf{k}}(s)\right\rangle+h_{\mathbf{k}} \int_{t_{0}}^{t} d s \chi_{\mathbf{k}}(t, s)+\left\langle f_{\mathbf{k}}^{0}\right\rangle,
\end{aligned}
$$

where the nonlinear damping rate

$$
\begin{aligned}
\eta_{\mathbf{k}}(t, s)= & -4 \sum_{\mathbf{p}} \sum_{\mathbf{q}} \delta(\mathbf{k}+\mathbf{p}+\mathbf{q}) K(\mathbf{k}, \mathbf{p}, \mathbf{q}) \\
& \times K(-\mathbf{p},-\mathbf{q},-\mathbf{k}) R_{-\mathbf{p}}(t, s) C_{-\mathbf{q}}(t, s)
\end{aligned}
$$

and

$$
\begin{aligned}
\chi_{\mathbf{k}}(t, s)= & 2 \sum_{\mathbf{p}} \sum_{\mathbf{q}} \delta(\mathbf{k}+\mathbf{p}+\mathbf{q}) K(\mathbf{k}, \mathbf{p}, \mathbf{q}) \\
& \times A(-\mathbf{p},-\mathbf{q},-\mathbf{k}) R_{-\mathbf{p}}(t, s) C_{-\mathbf{q}}(t, s) .
\end{aligned}
$$

From (11), see that the 2-time cumulant satisfies

$$
\begin{aligned}
& \left(\frac{\partial}{\partial t}+\nu_{0}(k) k^{2}\right) C_{\mathbf{k}}(t, \hat{t}) \\
& =\int_{t_{0}}^{t} d s\left[S_{\mathbf{k}}(t, s)+P_{\mathbf{k}}(t, s)+F_{\mathbf{k}}^{0}(t, s)\right] R_{-\mathbf{k}}(t, s) \\
& -\int_{t_{0}}^{t} d s\left[\eta_{\mathbf{k}}(t, s)+\pi_{\mathbf{k}}(t, s)\right] C_{-\mathbf{k}}(t, s) \\
& +\sum_{\mathbf{p}} \sum_{\mathbf{q}} \delta(\mathbf{k}+\mathbf{p}+\mathbf{q})\left\{K(\mathbf{k}, \mathbf{p}, \mathbf{q}) \tilde{K}_{-\mathbf{q},-\mathbf{p},-\mathbf{k}}^{(3)}(0,0,0)\right. \\
& \quad \times R_{-\mathbf{q}}(t, 0) R_{-\mathbf{p}}(t, 0) R_{-\mathbf{k}}(t, 0)+\left[2 K(\mathbf{k}, \mathbf{p}, \mathbf{q})\left\langle\zeta_{-\mathbf{q}}(t)\right\rangle\right. \\
& \left.\left.\quad+A(\mathbf{k}, \mathbf{p}, \mathbf{q}) h_{-\mathbf{q}}\right] \tilde{K}_{-\mathbf{p},-\mathbf{k}}^{(2)}(0,0) R_{-\mathbf{p}}(t, 0) R_{-\mathbf{k}}(t, 0)\right\},
\end{aligned}
$$


where we have employed the abbreviations

$$
\begin{aligned}
R_{\mathbf{k}}\left(t, t^{\prime}\right) & \equiv R_{\mathbf{k k}}\left(t, t^{\prime}\right), \\
C_{\mathbf{k}}\left(t, t^{\prime}\right) & \equiv C_{\mathbf{k}-\mathbf{k}}\left(t, t^{\prime}\right) .
\end{aligned}
$$

Here the bare noise is assumed to be white:

$$
F_{\mathbf{k}}^{0}(t, s)=\left\langle\hat{f}_{\mathbf{k}}^{0}(t) \hat{f}_{\mathbf{k}}^{0 *}(s)\right\rangle=F_{\mathbf{k}}^{0}(t) \delta(t-s),
$$

where $\delta$ is the Dirac delta function and $S$ is the nonlinear noise

$$
\begin{aligned}
S_{\mathbf{k}}(t, s)= & 2 \sum_{\mathbf{p}} \sum_{\mathbf{q}} \delta(\mathbf{k}+\mathbf{p}+\mathbf{q}) K(\mathbf{k}, \mathbf{p}, \mathbf{q}) \\
& \times K(-\mathbf{k},-\mathbf{p},-\mathbf{q}) C_{-\mathbf{p}}(t, s) C_{-\mathbf{q}}(t, s) .
\end{aligned}
$$

The $P$ and $\pi$ terms represent the inhomogeneous terms via coupling to the topography and mean-field

$$
\begin{aligned}
& P_{\mathbf{k}}(t, s)=\sum_{\mathbf{p}} \sum_{\mathbf{q}} \delta(\mathbf{k}+\mathbf{p}+\mathbf{q}) C_{-\mathbf{p}}(t, s) \\
& \quad \times\left[2 K(\mathbf{k}, \mathbf{p}, \mathbf{q})\left\langle\zeta_{-\mathbf{q}}(t)\right\rangle+A(\mathbf{k}, \mathbf{p}, \mathbf{q}) h_{-\mathbf{q}}\right] \\
& \quad \times\left[2 K(-\mathbf{k},-\mathbf{p},-\mathbf{q})\left\langle\zeta_{\mathbf{q}}(s)\right\rangle+A(-\mathbf{k},-\mathbf{p},-\mathbf{q}) h_{\mathbf{q}}\right], \\
& \pi_{\mathbf{k}}(t, s)=-\sum_{\mathbf{p}} \sum_{\mathbf{q}} \delta(\mathbf{k}+\mathbf{p}+\mathbf{q}) R_{-\mathbf{p}}(t, s) \\
& \quad \times\left[2 K(\mathbf{k}, \mathbf{p}, \mathbf{q})\left\langle\zeta_{-\mathbf{q}}(t)\right\rangle+A(\mathbf{k}, \mathbf{p}, \mathbf{q}) h_{-\mathbf{q}}\right] \\
& \quad \times\left[2 K(-\mathbf{p},-\mathbf{k},-\mathbf{q})\left\langle\zeta_{\mathbf{q}}(s)\right\rangle+A(-\mathbf{p},-\mathbf{k},-\mathbf{q}) h_{\mathbf{q}}\right] .
\end{aligned}
$$

The restart terms are calculated at time $t=t=T$ through the relationships

$$
\begin{aligned}
& \tilde{K}_{-\mathbf{p},-\mathbf{k}}^{(2)}(T, T)=K_{-\mathbf{p},-\mathbf{k}}^{(2) \operatorname{Dyn}}(T, T) \\
& \quad+\tilde{K}_{-\mathbf{p},-\mathbf{k}}^{(2)}(0,0) R_{-\mathbf{p}}(T, 0) R_{-\mathbf{k}}(T, 0) \\
& \tilde{K}_{-\mathbf{q},-\mathbf{p},-\mathbf{k}}^{(3)}(T, T, T)=K_{-\mathbf{q},-\mathbf{p},-\mathbf{k}}^{(3) \operatorname{Dyn}}(T, T, T) \\
& \quad+\tilde{K}_{-\mathbf{q},-\mathbf{p},-\mathbf{k}}^{(3)}(0,0,0) R_{-\mathbf{q}}(T, 0) R_{-\mathbf{p}}(T, 0) R_{-\mathbf{k}}(T, 0),
\end{aligned}
$$


where

$$
\begin{aligned}
& K_{-\mathbf{p},-\mathbf{k}}^{(2) \operatorname{Dyn}}(t, \hat{t}) \\
& =\int_{t_{0}}^{t} d s R_{-\mathbf{p}}(t, s) C_{-\mathbf{k}}(\dot{t}, s)\left[A(-\mathbf{p},-\mathbf{k}, \mathbf{k}+\mathbf{p}) h_{(-\mathbf{k}-\mathbf{p})}\right. \\
& \left.\quad+2 K(-\mathbf{p},-\mathbf{k}, \mathbf{k}+\mathbf{p})\left\langle\zeta_{(-\mathbf{k}-\mathbf{p})}(s)\right\rangle\right] \\
& \quad+\int_{t_{0}}^{t} d s R_{-\mathbf{k}}(\dot{t}, s) C_{-\mathbf{p}}(t, s)\left[A(-\mathbf{k},-\mathbf{p}, \mathbf{k}+\mathbf{p}) h_{(-\mathbf{k}-\mathbf{p})}\right. \\
& \left.\quad+2 K(-\mathbf{k},-\mathbf{p}, \mathbf{k}+\mathbf{p})\left\langle\zeta_{(-\mathbf{k}-\mathbf{p})}(s)\right\rangle\right]
\end{aligned}
$$

and

$$
\begin{aligned}
& \sum_{\mathbf{p}} \sum_{\mathbf{q}} \delta(\mathbf{k}+\mathbf{p}+\mathbf{q}) K(\mathbf{k}, \mathbf{p}, \mathbf{q}) K_{-\mathbf{q},-\mathbf{p},-\mathbf{k}}^{(3) \text { Dyn }}(t, t, \dot{t}) \\
& =\int_{t_{0}}^{t} d s S_{\mathbf{k}}(t, s) R_{-\mathbf{k}}(\dot{t}, s)-\int_{t_{0}}^{t} d s \eta_{\mathbf{k}}(t, s) C_{-\mathbf{k}}(\dot{t}, s) .
\end{aligned}
$$

The single time cumulant arises due to the fact that

$$
\frac{\partial C_{\mathbf{k}}(t, t)}{\partial t}=\lim _{t \rightarrow t}\left(\frac{\partial C_{\mathbf{k}}(t, \hat{t})}{\partial t}+\frac{\partial C_{\mathbf{k}}(t, \hat{t})}{\partial \hat{t}}\right)
$$

and $C_{\mathbf{k}}(t, \hat{t})=C_{\mathbf{k}}(\dot{t}, t)$ where for $t>t$ we have $R_{\mathbf{k}}(t, \hat{t})=0$. Thus 
the single time cumulant satisfies the equation

$$
\begin{aligned}
& \left(\frac{\partial}{\partial t}+2 \nu_{0}(k) k^{2}\right) C_{\mathbf{k}}(t, t) \\
& =2 \operatorname{Re} \int_{t_{0}}^{t} d s\left[S_{\mathbf{k}}(t, s)+P_{\mathbf{k}}(t, s)+F_{\mathbf{k}}^{0}(t, s)\right] R_{-\mathbf{k}}(t, s) \\
& -2 \operatorname{Re} \int_{t_{0}}^{t} d s\left[\eta_{\mathbf{k}}(t, s)+\pi_{\mathbf{k}}(t, s)\right] C_{-\mathbf{k}}(t, s) \\
& +2 \operatorname{Re} \sum_{\mathbf{p}} \sum_{\mathbf{q}} \delta(\mathbf{k}+\mathbf{p}+\mathbf{q})\left\{K(\mathbf{k}, \mathbf{p}, \mathbf{q}) \tilde{K}_{-\mathbf{q},-\mathbf{p},-\mathbf{k}}^{(3)}(0,0,0)\right. \\
& \quad \times R_{-\mathbf{q}}(t, 0) R_{-\mathbf{p}}(t, 0) R_{-\mathbf{k}}(t, 0)+\left[2 K(\mathbf{k}, \mathbf{p}, \mathbf{q})\left\langle\zeta_{-\mathbf{q}}(t)\right\rangle\right. \\
& \left.\left.\quad+A(\mathbf{k}, \mathbf{p}, \mathbf{q}) h_{-\mathbf{q}}\right] \tilde{K}_{-\mathbf{p},-\mathbf{k}}^{(2)}(0,0) R_{-\mathbf{p}}(t, 0) R_{-\mathbf{k}}(t, 0)\right\} .
\end{aligned}
$$

The equation for the response function takes a similar form:

$$
\begin{aligned}
& \left(\frac{\partial}{\partial t}+\nu_{0}(k) k^{2}\right) R_{\mathbf{k}}(t, \dot{t}) \\
& =-\int_{\dot{t}}^{t} d s\left[\eta_{\mathbf{k}}(t, s)+\pi_{\mathbf{k}}(t, s)\right] R_{\mathbf{k}}(s, t)
\end{aligned}
$$

with $R_{\mathbf{k}}(t, t)=1$. As we consider only white noise forcing, restart terms are not required for the propagator $R,(31)$. This would not be the case for coloured noise.

\section{Restart terms}

The QDIA, like other non-Markovian closure theories, may be computationally expensive for long time integrations because of the time-history integrals which need to be evaluated between the initial 
and final times. The cumulant update restart procedure consists of integrating the QDIA forward for a time, calculating the 2- and 3point terms at this time and then using these in the new initial conditions for further integration. In principle, knowledge of all higher order cumulants should be available, but to be consistent with the approximations of the QDIA only the 2- and 3-point cumulants are needed.

The 3-point cumulant is effectively the homogeneous component of the closure equations, whereas the 2-point terms arise due to the inhomogeneity produced by the presence of topography and mean field. Thus we write the tendency of the 2-time cumulant as being composed of 2- and 3-point terms:

$$
\begin{aligned}
& \left(\frac{\partial}{\partial t}+\nu_{0}(k) k^{2}\right) C_{k}(t, \hat{t}) \\
& =\sum_{\mathbf{p}} \sum_{\mathbf{q}} \delta(\mathbf{k}+\mathbf{p}+\mathbf{q}) A(\mathbf{k}, \mathbf{p}, \mathbf{q}) C_{-\mathbf{p}-\mathbf{k}}(t, \hat{t}) h_{-\mathbf{q}} \\
& +\sum_{\mathbf{p}} \sum_{\mathbf{q}} \delta(\mathbf{k}+\mathbf{p}+\mathbf{q}) K(\mathbf{k}, \mathbf{p}, \mathbf{q})\left[\left\langle\zeta_{-\mathbf{p}}(t)\right\rangle C_{-\mathbf{q}-\mathbf{k}}(t, \hat{t})\right. \\
& \left.\quad+C_{-\mathbf{p}-\mathbf{k}}(t, \hat{t})\left\langle\zeta_{-\mathbf{q}}(t)\right\rangle+\left\langle\hat{\zeta}_{-\mathbf{p}}(t) \hat{\zeta}_{-\mathbf{q}}(t) \hat{\zeta}_{-\mathbf{k}}(\hat{t})\right\rangle\right] \\
& +\int_{t_{0}}^{t} d s F_{\mathbf{k}}^{0}(t, s) R_{-\mathbf{k}}(\hat{t}, s)
\end{aligned}
$$

where we define the 2-point cumulant

$$
C_{\mathbf{k}-\mathbf{p}}(t, \hat{t})=\left\langle\hat{\zeta}_{-\mathbf{k}}(t) \hat{\zeta}_{-\mathbf{p}}(\hat{t})\right\rangle,
$$

and 3-point cumulant

$$
\left\langle\hat{\zeta}_{-\mathbf{k}}(t) \hat{\zeta}_{-\mathbf{p}}(t) \hat{\zeta}_{-\mathbf{q}}(t)\right\rangle
$$

respectively. 
If we follow the argument of Rose [3] and suppose that the initial 2 - and 3-point cumulants are non-zero then we are confronted with the in principle non-vanishing of other higher order cumulants $(\geq 3)$. These higher order cumulants arise due to the nonlinearity in the expansion parameter $\lambda$ (see equation (40)). To see this consider the inhomogeneous DIA

$$
\begin{aligned}
& \left(\frac{\partial}{\partial t}+\nu_{0}(k) k^{2}\right) C_{\mathbf{k}}(t, \hat{t})= \\
& \int_{t_{0}}^{t} d s\left[S_{\mathbf{k}}(t, s)+P_{\mathbf{k}}(t, s)+F_{\mathbf{k}}^{0}(t, s)\right] R_{-\mathbf{k}}(\dot{t}, s) \\
& -\int_{t_{0}}^{t} d s\left[\eta_{\mathbf{k}}(t, s)+\pi_{\mathbf{k}}(t, s)\right] C_{-\mathbf{k}}(\dot{t}, s) .
\end{aligned}
$$

When $\zeta_{\mathbf{k}}(0)$ is Gaussian we write the nonlinear noise $S_{\mathbf{k}}$, nonlinear damping $\eta_{\mathbf{k}}$ and inhomogeneous contributions, $P_{\mathbf{k}}$ and $\pi_{\mathbf{k}}$ (which are composed of $\eta_{\mathbf{p}}, \eta_{\mathbf{q}}, \chi_{\mathbf{p}}$ and $\chi_{\mathbf{q}}$ terms, while the evolution equation for $\left\langle\zeta_{\mathbf{k}}\right\rangle$ consists of $\eta_{\mathbf{k}}, \chi_{\mathbf{k}}$ components) as a power series in $\lambda$, that is,

$S=\alpha_{n} \lambda^{n}, \quad \eta=\beta_{n} \lambda^{n}, \quad P=\gamma_{n} \lambda^{n} \quad \pi=\sigma_{n} \lambda^{n}, \quad n=2,3, \ldots,(36)$

where $\alpha_{n}, \beta_{n}, \gamma_{n}$ and $\sigma_{n}$ consist of finite products of $R$ and $C$. It is the dependence of these terms on the exact $R$ and $C$ that constitutes a propagator renormalised expansion, where the DIA is truncated at $\mathcal{O}\left(\lambda^{2}\right)$.

See that for non-vanishing 2- and 3-point cumulants, (36) convert to multiple power series in $\lambda$ and all non-vanishing initial cumulants. Hence in order to be consistent with the approximations of the DIA we need only consider the 2- and 3-point cumulants. Thus the inhomogeneous cumulant update DIA equations are those in the preceding section, where we integrate up to some time 
$t=t=T$ and calculate the 2 - and 3-point cumulants through the relations (25-28). The procedure is repeated as often as needed simply by replacing $\tilde{K}_{-\mathbf{p},-\mathbf{k}}^{2}(0,0)$ and $\tilde{K}_{-\mathbf{q},-\mathbf{p},-\mathbf{k}}^{3}(0,0,0)$ with the quantities $\tilde{K}_{-\mathbf{p},-\mathbf{k}}^{2}(T, T)$ and $\tilde{K}_{-\mathbf{q},-\mathbf{p},-\mathbf{k}}^{3}(T, T, T)$.

\section{$5 \quad$ Numerical methods}

The numerical approach that we have followed mirrors that of the original work of Kraichnan [6] and subsequently used for investigations of homogeneous closure theories $[4,5,7,8]$. The forward time step is achieved via a predictor-corrector scheme while the time-history integrals in the cumulant and mean-field equations are discretized by the trapezoidal rule. The truncation in wavenumber is to $k=3$ which, although severe, still allows sufficient degrees of freedom so that the system is mixing [9]. In the second order closure considered, three interacting simple shear waves result in the generation of triads in wavenumber space as evident in (8). The closure and DNS models are formulated for discrete spectra on the doubly periodic domain and run over the whole wavenumber space allowing unambiguous comparison. Further efficiency is gained by running over the half-space and invoking conjugacy thereby reducing the number of interaction coefficients by half and gaining a doubling in computational speed. The $k=3$ case presented (see Figure 1) has 28 components which due to degeneracy reduce to six distinct 2 -point cumulant values within the closures. We also have four sets of 360 non-zero interaction coefficients to be summed over which for increasing time represents a distinct computational challenge even when employing the restart procedure. 


\section{Comparison of QDIA and CUQDIA with DNS}

We have employed typical meteorological time and space scales with a length scale of $a / 2$, where $a=6.37122 \times 10^{6} \mathrm{~m}$ is the earth's radius and a time scale $(\sqrt{2} \Omega)^{-1}$, where $\Omega=7.292 \times 10^{-5} \mathrm{~s}^{-1}$ is the earth's angular velocity. The non-dimensional time step used is 1.1136 corresponding to $1 / 8$ th days, while the more general $\nu_{0}(k)$ is replaced by $\hat{\nu}=1.8579 \times 10^{-2}$ the nondimensional viscosity coefficient. The variance of the white noise forcing and mean-field forcing are derived by canonical equilibrium theory and are

$$
\begin{aligned}
& F_{\mathbf{k}}^{0}=2 \hat{\nu}_{0}(k) k^{2} C_{\mathbf{k}}^{e q}, \\
& \left\langle\zeta_{\mathbf{k}}^{e q}\right\rangle=-b_{2} h_{\mathbf{k}} C_{\mathbf{k}}^{e q},
\end{aligned}
$$

where

$$
C_{\mathbf{k}}^{e q}=\frac{k^{2}}{a_{2}+b_{2} k^{2}},
$$

thus the closures are forced to asymptote to the equilibrium solution. In Figures 1 and 2 we have chosen $a_{2}=-5.969 \times 10^{5}$ and $b_{2}=$ $7.444 \times 10^{5}$ with $a_{1}=4.842 \times 10^{4}$ and $b_{1}=2.511 \times 10^{3}$ used in the generation of the initial $C_{\mathbf{k}}(0,0)$. The mean-field is initially zero and is "spun up" via the interaction of the fluctuating field and the topography which is chosen so that at equilibrium the mean and fluctuating fields are of equal magnitude. The initial $C_{\mathbf{k}}(0,0)$ is chosen in order to ensure non-trivial behaviour in the early period of evolution. The DNS results represent an average of 5000 realizations whose initial conditions at $t=0$ have real and imaginary parts with a joint Gaussian distribution. The CUQDIA results are for restarts at $t=t=T=20 \delta t$ and demonstrate excellent agreement with the QDIA results (up to 6 significant figures). Note: at equilibrium 

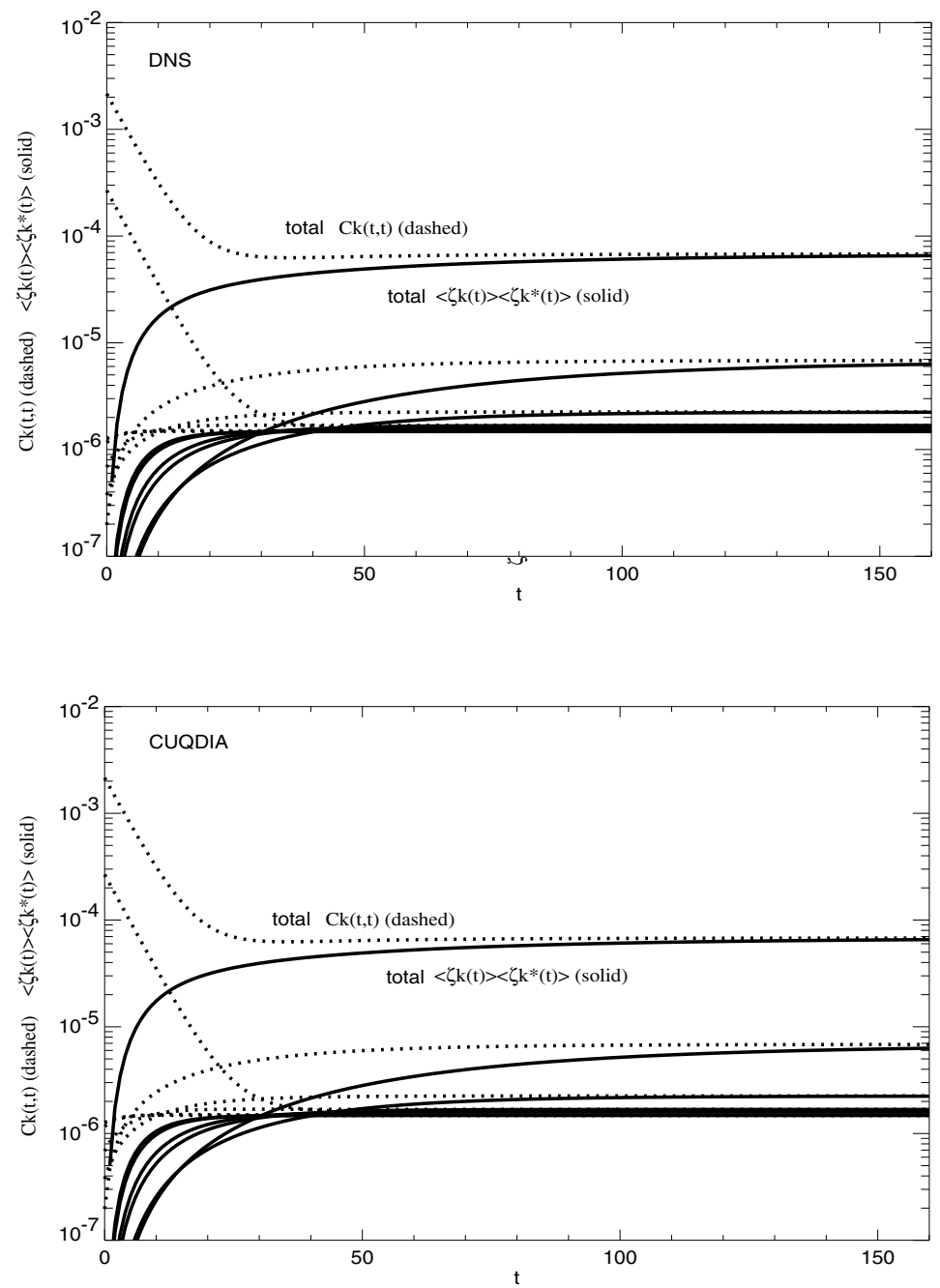

FIgURE 1: A comparison of QDIA and CUQDIA results with restarts every $t=t=T=20 \delta t$. The parameters are detailed in Figure 2 . 


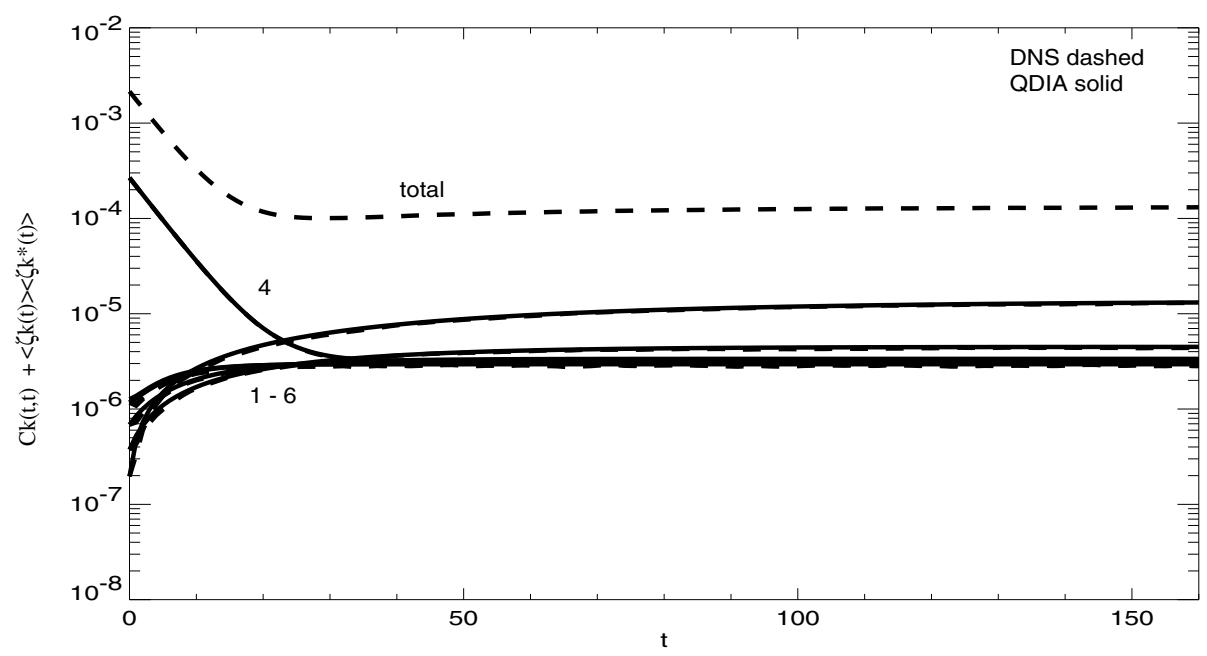

FIGURE 2: A comparison of QDIA and DNS results for the total fields: $\hat{\nu}=1.8579 \times 10^{-2} ; C_{\mathbf{k}}^{e q}=\frac{k^{2}}{a_{2}+b_{2} k^{2}}$; initial twice enstrophies $C_{\mathbf{k}}(0,0)=10^{-2} \times \frac{k^{2}}{a_{1}+b_{1} k^{2}}$, where $C_{(2,1)}=2.7622 \times 10^{-4}$; twice enstrophies forcing $F_{\mathbf{k}}^{0}=2 \hat{\nu} k^{2} C_{\mathbf{k}}^{e q}$; topographic amplitude squared $\left|h_{\mathbf{k}}\right|^{2}=\frac{a_{2}+b_{2} k^{2}}{b_{2}^{2} k^{2}} ;\left\langle\zeta_{\mathbf{k}}^{e q}\right\rangle=-b_{2} h_{\mathbf{k}} C_{\mathbf{k}}^{e q} ;$ initial mean-field $\left\langle\zeta_{\mathbf{k}}(0)\right\rangle=0 ;$ mean-field forcing $\left\langle f_{\mathbf{k}}^{0}\right\rangle=\hat{\nu} k^{2}\left\langle\zeta_{\mathbf{k}}^{e q}\right\rangle ; a_{1}=4.842 \times 10^{4} ; b_{1}=2.511 \times$ $10^{3} ; a_{2}=-5.969 \times 10^{5} ; b_{2}=7.444 \times 10^{5} ; \delta t=1 / 8$ days 
the integral terms of the closure vanish and the restart becomes exact. The comparison of the total field with DNs (5000 realizations) also shows close agreement apart from slight variation in the regime where transients are dominant as is standard for DIA $[6,7,8]$ based closures. The reduction in computational cost is great with the QDIA calculation taking several hours on a Cray J90 while the similar CUQDIA finished in only a few minutes. Finally, note that due to degeneracy only the first six modes need be presented in Figures 1 and 2 .

\section{Conclusion}

We have presented results for a tractable closure theory for inhomogeneous flow over mean topography with and without non-Gaussian restarts. The model has been shown to be in good agreement with DNS calculations. The restart calculation has been shown to dramatically reduce the computational cost of the calculation while again showing close agreement to both DNS and QDIA results. In future studies we plan to examine the performance of the QDIA and CUQDIA closures at higher resolution.

Acknowledgment: TJO gratefully acknowledges postgraduate research scholarships from the School of Mathematical Sciences, Monash University, the Co-operative Research Centre for Southern Hemisphere Meteorology and CSIRO Atmospheric Research. 


\section{A 2-point restart terms}

Suppose we expand $\zeta_{\mathbf{k}}$ in a perturbation series

$$
\zeta_{\mathbf{k}}(t)=\zeta_{\mathbf{k}}^{0}(t)+\lambda \zeta_{\mathbf{k}}^{1}(t)+\cdots
$$

To zeroth order we have from (5)

$$
\left(\frac{\partial}{\partial t}+\nu_{0}(k) k^{2}\right) \hat{\zeta}_{\mathbf{k}}^{0}(t)=\hat{f}_{\mathbf{k}}^{0}(t)+\delta\left(t-t_{0}\right) \hat{\zeta}_{\mathbf{k}}^{0}\left(t_{0}\right),
$$

with solution

$$
\hat{\zeta}_{\mathbf{k}}^{0}(t)=R_{\mathbf{k}}\left(t, t_{0}\right) \hat{\zeta}_{\mathbf{k}}^{0}\left(t_{0}\right)+\int_{t_{0}}^{t} d s R_{\mathbf{k}}^{0}(t, s) \hat{f}_{\mathbf{k}}^{0}(s) .
$$

To order $\lambda$ we have

$$
\begin{aligned}
& \left(\frac{\partial}{\partial t}+\nu_{0}(k) k^{2}\right) \hat{\zeta}_{\mathbf{k}}^{1}=\sum_{\mathbf{p}} \sum_{\mathbf{q}} \delta(\mathbf{k}+\mathbf{p}+\mathbf{q}) A(\mathbf{k}, \mathbf{p}, \mathbf{q}) \hat{\zeta}_{-\mathbf{p}}^{0} h_{-\mathbf{q}} \\
& +\sum_{\mathbf{p}} \sum_{\mathbf{q}} \delta(\mathbf{k}+\mathbf{p}+\mathbf{q}) K(\mathbf{k}, \mathbf{p}, \mathbf{q})\left[\left\langle\zeta_{-\mathbf{p}}\right\rangle \hat{\zeta}_{-\mathbf{q}}^{0}+\left\langle\zeta_{-\mathbf{q}}\right\rangle \hat{\zeta}_{-\mathbf{p}}^{0}\right. \\
& \left.\quad+\hat{\zeta}_{-\mathbf{p}}^{0} \hat{\zeta}_{-\mathbf{q}}^{0}-\left\langle\hat{\zeta}_{-\mathbf{p}}^{0} \hat{\zeta}_{-\mathbf{q}}^{0}\right\rangle\right]+\delta\left(t-t_{0}\right) \hat{\zeta}_{\mathbf{k}}^{1}\left(t_{0}\right)
\end{aligned}
$$

Thus

$$
\hat{\zeta}_{\mathbf{k}}^{1}(t)=\hat{\zeta}_{\mathbf{k}}^{1(\mathrm{Dyn})}(t)+R_{\mathbf{k}}^{0}\left(t, t_{0}\right) \hat{\zeta}_{\mathbf{k}}^{1}\left(t_{0}\right)
$$

where

$$
\begin{aligned}
& \hat{\zeta}_{\mathbf{k}}^{1(\mathrm{Dyn})}(t)=\int_{t_{0}}^{t} d s R_{\mathbf{k}}^{0}(t, s) \\
& \times\left\{\sum_{\mathbf{p}} \sum_{\mathbf{q}} \delta(\mathbf{k}+\mathbf{p}+\mathbf{q}) A(\mathbf{k}, \mathbf{p}, \mathbf{q}) \hat{\zeta}_{-\mathbf{p}}^{0}(s) h_{-\mathbf{q}}\right. \\
& \quad+\sum_{\mathbf{p}} \sum_{\mathbf{q}} \delta(\mathbf{k}+\mathbf{p}+\mathbf{q}) K(\mathbf{k}, \mathbf{p}, \mathbf{q})\left[\left\langle\zeta_{-\mathbf{p}}(s)\right\rangle \hat{\zeta}_{-\mathbf{q}}^{0}(s)\right. \\
& \left.\left.\quad+\left\langle\zeta_{-\mathbf{q}}(s)\right\rangle \hat{\zeta}_{-\mathbf{p}}^{0}(s)+\hat{\zeta}_{-\mathbf{p}}^{0}(s) \hat{\zeta}_{-\mathbf{q}}^{0}(s)-\left\langle\hat{\zeta}_{-\mathbf{p}}^{0}(s) \hat{\zeta}_{-\mathbf{q}}^{0}(s)\right\rangle\right]\right\}
\end{aligned}
$$


Finally

$$
\begin{aligned}
& C_{\mathbf{k}-\mathbf{l}}^{1}(t, \hat{t})=\left\langle\hat{\zeta}_{\mathbf{k}}^{1(\text { Dyn })}(t) \hat{\zeta}_{-\mathbf{l}}^{0}(\hat{t})\right\rangle+\left\langle\hat{\zeta}_{\mathbf{k}}^{0}(t) \hat{\zeta}_{-\mathbf{l}}^{1(\text { Dyn })}(\dot{t})\right\rangle \\
& +R_{\mathbf{k}}^{0}\left(t, t_{0}\right)\left\langle\hat{\zeta}_{\mathbf{k}}^{1}\left(t_{0}\right) \hat{\zeta}_{-\mathbf{l}}^{0}(t)\right\rangle+R_{-\mathbf{l}}^{0}\left(\dot{t}, t_{0}\right)\left\langle\hat{\zeta}_{\mathbf{k}}^{0}(t) \hat{\zeta}_{-\mathbf{l}}^{1}\left(t_{0}\right)\right\rangle \\
& =\left\langle\hat{\zeta}_{\mathbf{k}}^{1(\text { Dyn })}(t) \hat{\zeta}_{-\mathbf{l}}^{0}(\hat{t})\right\rangle+\left\langle\hat{\zeta}_{\mathbf{k}}^{0}(t) \hat{\zeta}_{-\mathbf{l}}^{1(\text { Dyn })}(\hat{t})\right\rangle \\
& +R_{\mathbf{k}}^{0}\left(t, t_{0}\right) R_{-\mathbf{l}}^{0}\left(\dot{t}, t_{0}\right)\left\langle\hat{\zeta}_{\mathbf{k}}^{1}\left(t_{0}\right) \hat{\zeta}_{-\mathbf{l}}^{0}\left(t_{0}\right)\right\rangle \\
& +R_{\mathbf{k}}^{0}\left(t, t_{0}\right) R_{-\mathbf{l}}^{0}\left(\dot{t}, t_{0}\right)\left\langle\hat{\zeta}_{\mathbf{k}}^{0}\left(t_{0}\right) \hat{\zeta}_{-\mathbf{l}}^{1}\left(t_{0}\right)\right\rangle \\
& =\left\langle\hat{\zeta}_{\mathbf{k}}^{1(\text { Dyn })}(t) \hat{\zeta}_{-\mathbf{l}}^{0}(t)\right\rangle+\left\langle\hat{\zeta}_{\mathbf{k}}^{0}(t) \hat{\zeta}_{-\mathbf{l}}^{1(\text { Dyn })}(t)\right\rangle \\
& +R_{\mathbf{k}}^{0}\left(t, t_{0}\right) R_{-\mathbf{l}}^{0}\left(\dot{t}, t_{0}\right) C_{\mathbf{k}-\mathbf{l}}\left(t_{0}, t_{0}\right)
\end{aligned}
$$

where we have assumed that only the initial fields yield correlation for off-diagonal elements. Thus the 2-point restart terms are those given by (25) and (27).

\section{References}

[1] J. S. Frederiksen, Subgrid-scale parameterizations of eddy-topographic force, eddy viscosity, and stochastic backscatter for flow over topography, J. Atmos. Sci., Volume 56, pages 1481-1494, 1999. C570, C574, C575

[2] G. Holloway, Representing topographic stress for large-scale ocean models, J. Phys. Oceanogr., volume 22, pages 1033-1046, 1992. C570

[3] H. A. Rose, An efficient non-Markovian theory of non-equilibrium dynamics, Physica 14D , pages 216-226, 1985. C571, C581

[4] J. S. Frederiksen, A. G. Davies, and R. C. Bell. Closure theories with non-Gaussian restarts for truncated 
two-dimensional turbulence, Phys. Fluids, volume 6, pages 3153-3163, 1994. C571, C582

[5] J. S. Frederiksen and A. G.Davies, Dynamics and spectra of cumulant update closures for two-dimensional turbulence, Geophys. Astrophys. Fluid Dynamics, volume 92, pages 197-231, 2000 C571, C582

[6] R. H. Kraichnan, Decay of isotropic turbulence in the direct-interaction approximation, Phys. Fluids, volume 7, pages 1030-1048, 1964 C582, C586

[7] J. S. Frederiksen and R. C.Bell, Simulations and closure theory for interacting barotropic waves, CTAC-87, Elsevier Science Publications B.V. (North Holland), pages 207-216, 1988 C582, C586

[8] J. S. Frederiksen and A. G.Davies, Performance of integro-differential closure equations for two-dimensional turbulence, CTAC-95, World Scientific Publishing Co, pages 319-326, 1996 C582, C586

[9] L. C. Kells and S. A. Orszag, Randomness of low-order models of two-dimensional inviscid dynamics, Phys. Fluids, volume 21, pages 162-168, 1978 C582 